\title{
Enquête sur l'utilisation des anesthésiques locaux par les chirurgiens dentistes exerçant en Alsace
}

\author{
A survey of local anaesthetic use among dental practitioners in Alsace (France)
}

\author{
ALINE VAN DER AUWERA ${ }^{1}$, ABDESSAMAD BOUKARI ${ }^{1}$, NICOLAS MEYER ${ }^{2}$, SOPHIE BAHI', AHMED FEKI'
}

\begin{abstract}
RÉSUMÉ
Objectifs : L'objectif de cette enquête était d'identifier les pratiques et les usages des anesthésiques locaux lors des différents types d'actes par les chirurgiens dentistes exerçant en Alsace.

Matériels et méthodes : L'ensemble des praticiens exerçant en Alsace (1500) ont reçu un questionnaire de quatre pages par différents moyens. 311 questionnaires ont été renvoyés $(20.7 \%)$ et 308 ont été retenus. Les réponses ont été enregistrées dans un tableau Excel après anonimisation. L'enquête a été conduite durant les mois de mai et juin 2005.

Résultats : La majorité des praticiens (230/290 = $79.3 \%$ [74.2 - 83.8]) utilisent une seule molécule anesthésique (majoritairement l'articaïne, la lidocaïne en second). Le choix de la molécule anesthésique utilisée est le même dans les différentes situations physiologiques ou pathologiques proposées dans le questionnaire. Face à un patient en bonne santé, la majorité des praticiens utilisent des anesthésiques locaux contenant de l'adrénaline $(249 / 291=85.6 \%$ [81.0 - 89.4]). Le second vasoconstricteur le plus fréquemment choisi est la noradrénaline $(30 / 291=10.3 \%$ [7.1-14.4]). Le choix du vasoconstricteur ne change pas de façon significative en fonction de l'état physiologique ou pathologique des patients. Toutefois $23-25 \%$ des praticiens s'abstiennent de choisir un vasoconstricteur lorsque le patient présente une situation physiologique ou pathologique particulière. $59.9 \%$ (176/294 = $59.9 \%$ [54.0 - 65.5]) des praticiens utilisent un anesthésique de contact avant l'infiltration. L'anesthésie transcorticale est pratiquée par $53.9 \%$ (159/295 = $53.9 \%$ [48.0 - 59.7]) des praticiens, dans $76.7 \%$ des cas $(122 / 159=76.7$ [69.4 - 83.1]) avec des vasoconstricteurs, mais seulement $44.7 \%$ $(71 / 159=44.7 \%$ [36.8 - 52.7]) d'entre eux ont recours à du matériel spécifique pour ce type d'injection. Pour réaliser des actes courants de soins ou de chirurgie, l'anesthésie par infiltration para-apicale est la technique la plus couramment pratiquée au maxillaire et dans les secteurs antérieurs à la mandibule (environ $80 \%$ ), tandis que dans les secteurs postérieurs de la mandibule, les praticiens réalisent les différentes techniques : paraapicales, locorégionales et intraligamentaires. Les praticiens qui réalisent de la chirurgie implantaire ont recours à des anesthésies para-apicales en grande majorité (entre 50 et $71 \%$ en fonction des secteurs.) $88 \%$ des praticiens ont étés confrontés au moins une fois à une complication après une anesthésie locale. Les plus fréquemment citées sont les hématomes $(271 / 289=93.8 \%$ [90.3 - 96.3]) et les réactions vagales $(206 / 293=$ $70.3 \%$ [64.7 - 75.5]).

Conclusions : La grande majorité des praticiens exerçant en Alsace choisissent d'utiliser des solutions anesthésiques contenant de l'articaïne et de l'adrénaline pour la plupart de leurs actes et quelle que soit la situation physiologique ou pathologique du patient. L'anesthésie para-apicale est la technique la plus pratiquée. La plupart des praticiens ont eu l'expérience d'une complication classique après une anesthésie locale. Med Buccale Chir Buccale 2007; 13 : 187-198.
\end{abstract}

mots clés: Anesthésiques locaux, vasoconstricteurs, infiltration para-apicale, anesthésie transcorticale, anesthésie locorégionale, anesthésie intraligamentaire, hématome, réaction vagale.

\footnotetext{
1. Département de Médecine et Chirurgie buccales, Hôpitaux universitaires de Strasbourg

2. Département d'Informatique médicale, Hôpitaux universitaires de Strasbourg
}

Demande de tirés à part:

Aline Van der Auwera 1, rue de Kembs 68510 Sierentz France thesevda@yahoo.fr 
médecine buccale chirurgie buccale

VOL. $13, \mathrm{~N}^{\circ} 4$ 2007

page 188

\section{SUMMARY}

Objective: The aim of this study was to identify local anesthetic practices in dental care in the region of Alsace, East of France.

Materials and methods: All dentists in Alsace (1500) received a four pages questionnaire by several methods. 311 questionnaires were received (20.7\%) and 308 were included in an excel database after quality control and anonymization. The survey was conducted in may-june 2005.

Results: Most of the dentists (230/290 = 79.3\% [74.2 - 83.8]) used only one molecule (mostly articaine, with lidocaine in second rank). The choice of anaesthetic remained the same across a variety of physiological and pathological circumstances. In healthy patients, epinephrine remained the preferred vasoconstrictor (249/291 = 85.6\% [81.0 - 89.4]) followed by norepinephrine (30/291 = 10.3\% [7.1-14.4]) on second rank. The choice of vasoconstrictor did not substantially change across various clinical conditions. However 23-25\% of practitioners abstained to chose a vasoconstrictor in variety of physiological and pathological circumstances. Topical mucosal application or anaesthetic gel was chosen by 59.9\% (176/294 = 59.9\% [54.0 - 65.5]) of practitioners. Transcortical anaesthesia was reported by $53.9 \%(159 / 295=53.9 \%$ [48.0 - 59.7]) of the practitioners - 76.7\% (122/159 = 76.7\% [69.4 - 83.1]) with vasoconstrictor - and 44.7\% (71/159 = 44.7\% [36.8 - 52.7]) of them using specific injector. In common dental care and surgery, para-apical anesthesia is the predominant technique for the maxilla and anterior sector of mandibula (about 80\%) while the posterior sector of mandibula para-apical, locoregional and intraligamental techniques were balanced. Regarding all sectors, para-apical anesthesia is the preferred technique (between 50 and 71\%) among practitioners declared to place implants. Complications were experienced by $88 \%$ of the practitioners. Haematoma (271/289 = 93.8\% [90.3 - 96.3]), autonomous vagal reaction (206 / $293=70.3 \%$ [64.7 - 75.5]) are mostly describes.

Conclusions: Dental practitioners in Alsace used mainly articaine with epinephrine as their preferred anaesthetic solution for most of the dental procedures across the variety of their patients. Para-apical anaesthesia remains the preferred technique. Classical complications of local anaesthesia are experienced by most of the practitioners. Med Buccale Chir Buccale 2007; 13 : 187-198

key words: Local anesthetics, vasoconstrictors, transcortical anesthesia, para-apical anesthesia, locoregional anesthesia, intraligamental anesthesia, haematoma, autonomous vagal reaction.

En France, de nombreuses molécules sont disponibles dans les solutions anesthésiques locales : l'articaïne, l'aptocaïne, la mépivacaïne, la lidocaïne, la procaïne et la prilocaïne. Elles sont disponibles avec ou sans vasoconstricteurs (adrénaline, noradrénaline, corbadrine ou lévonoréphédrine).

Les praticiens choisissent la solution anesthésique locale en fonction de la situation physiologique ou pathologique du patient et du type et de la localisation de l'acte à réaliser.

L'objectif de cette enquête était de décrire l'utilisation des anesthétiques locaux en odontologie par les praticiens exerçant en Alsace (France.) La population alsacienne est de 1794000 habitants (recensement de 2004), soignés par 1500 chirurgiens-dentistes (soit 1196 habitants par dentistes).
Une étude similaire a précédemment été conduite en France en 2000 [1] ainsi qu'au Royaume Uni [2], en Allemagne ${ }^{[3]}$ et au Brésil [4].

\section{MATERIEL ET METHODES}

L'enquête a été conduite aux mois de mai et juin 2005. Le questionnaire était anonyme et comprenait quatre pages de questions-réponses à cocher. Le remplissage nécessitait 5 à 10 minutes. Pour encourager les réponses, les questionnaires ont étés distribués par e-mail et fax. De plus, dans le Haut-Rhin (509 praticiens), tous les praticiens ont reçu le questionnaire par courrier postal. Dans le Bas-Rhin (991 praticiens) le questionnaire était transmis par courrier postal lorsque le praticien ne possédait ni fax, ni mail. 311 questionnaires 
on été renvoyés (20.7\%). Après avoir contrôlé l'absence de duplication, trois questionnaires ont été exclus : I'un avait été complété par un praticien exerçant en dehors de l'Alsace et deux n'avaient été complétés que pour les données démographiques. Les 308 questionnaires restants ont été rendus anonymes et les réponses enregistrées dans un tableau Excel. Les résultats ont été analysés en fonction de statistiques décrivant des variables qualitatives. Les proportions sont données avec un intervalle de confiance de $95 \%$. Le questionnaire était structuré de la façon suivante :

- Informations démographiques : ville/village d'exercice, le mode d'exercice, la spécialité et la présence ou non d'au moins une assistante.

- Le choix de la molécule anesthésique en fonction des situations physiologiques ou pathologiques des patients : adulte sain, enfant, femme enceinte, intoxication alcoolo-tabagique, patient sous traitement psychotrope, pathologies cardiovasculaires et handicaps mentaux.

- Le choix du vasoconstricteur en fonction des situations physiologiques ou pathologiques des patients : adulte sain, enfant, femme enceinte, patient âgé, diabète, pathologie cardiovasculaire, pathologie mentale, patient sous traitement psychotrope.

- Utilisation ou pas d'anesthésie topique.

- Les techniques anesthésiques utilisées lors des soins, de la chirurgie et de la chirurgie implantaire dans les différents secteurs (maxillaire, mandibule, antérieure ou postérieure) avec, comme choix proposés: la technique para-apicale, locorégionale, intraligamentaire ou transcorticale.

- Les complications consécutives à une anesthésie locale auxquels les praticiens avaient été confrontés au moins une fois.

\section{RESULTATS}

\section{Données démographiques}

Sur les 308 questionnaires analysés, $58 \%$ provenaient du Haut-Rhin et $36 \%$ du Bas-Rhin, $6 \%$ ne comprenaient pas d'indication de région exploitable. La répartition des praticiens en Alsace est de $34 \%$ dans le Haut-Rhin et $66 \%$ dans le Bas-Rhin. La différence provient de la stratégie de distribution des questionnaires dans les deux départements (questionnaire envoyés plusieurs fois par différents moyens dans le Haut-Rhin). Les praticiens ayant répondu ont une durée d'exercice équitablement répartie par rapport à la population réelle (de 1 à 38 ans).

$71.7 \%(205 / 286=71.7 \%$ [66.1 - 76.8]) des praticiens exercent dans une agglomération de plus de 5000 habitants. La majorité des praticiens travaillent seuls dans leur cabinet $(54.1 \%)$ tandis que $39.1 \%$ (119/306=39.1\% [33.6 - 44.9]) exerçent en association de plusieurs praticiens ; les autres cas représentent les praticiens salariés ou remplaçants. $93.1 \%(283 / 304=93.1 \%$ [89.6 95.7]) des praticiens emploient au moins une assistante dentaire et seulement $6.9 \%(21 / 304=$ $6.9 \%$ [4.3 - 10.4]) travaillent sans assistante. La majorité des praticiens se décrivent comme omnipraticiens $(85.6 \%)(262 / 306=85.6 \%$ [81.2 $89.4]), 4.9 \%(15 / 306=4.9 \%$ [2.8 - 8.0]) des praticiens considèrent que leur activité est orientée vers l'implantologie, les autres spécialisations (ODF, chirurgie, parodontologie, pédodontie, endodontie ou la combinaison de plusieurs de ces disciplines) représentent moins de $2 \%$ des réponses.

\section{Choix de l'anesthésique}

En général, $79.3 \%(230 / 290=79.3 \%$ [74.2 83.8]) des praticiens ne disposent que d'une seule molécule anesthésique au cabinet. Seulement $17.6 \%(51 / 290=17.6 \%$ [13.4 - 22.5]) en possèdent deux, $2.8 \%(8 / 290=2.8 \%[1.2-5.4])$ trois et un seul praticien quatre. Comme décrit dans le tableau 1, l'articaïne est la molécule anesthésique la plus utilisée. Lorsque deux molécules sont disponibles, le second choix des praticiens se porte sur la lidocaïne.

Quelles que soient les situations physiologiques ou pathologiques rencontrées chez les patients, le choix de la molécule anesthésique rapporté par les praticiens change peu.

Le tableau 2 rapporte les pourcentages des praticiens utilisant la même molécule anesthésique, majoritairement l'articaïne, pour anesthésier un patient sain et un patient présentant l'une des conditions physiologique ou pathologique proposées dans le questionnaire. L'enquête révèle médecine

buccale

chirurgie

buccale

VOL. $13, \mathrm{~N}^{\circ} 4$ 2007

page 189 
Tableau 1 : Molécules utilisées pour l'anesthésie locale par les praticiens en Alsace (France) (100\% = 308 chirurgiens-dentistes) (pourcentages).

Choice of local anaesthetics in dental practice in Alsace (France) $(100 \%=308$ dentists) (percentage).

médecine

buccale

chirurgie

buccale

VOL. $13, \mathrm{~N}^{\circ} 4$ 2007

page 190

\begin{tabular}{|c|c|c|c|c|c|c|c|c|}
\hline $\begin{array}{l}\text { Patient } \\
\text { condition }\end{array}$ & 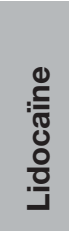 & 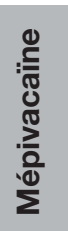 & 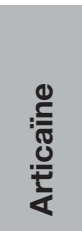 & 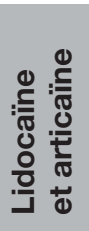 & 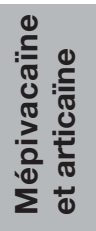 & 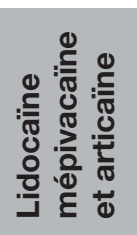 & 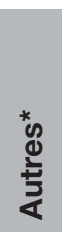 & $\begin{array}{l}0 \\
0 \\
0 \\
0 \\
0 \\
0 \\
0 \\
0\end{array}$ \\
\hline Adulte sain & 4.9 & 1.3 & 76.6 & 6.5 & 1.6 & 1.0 & 4.8 & 4.2 \\
\hline Enfant sain & 6.8 & 2.3 & 75.3 & 6.5 & 0.6 & 0.3 & 2.6 & 5.8 \\
\hline Femme enceinte & 5.8 & 2.3 & 72.7 & 1.9 & 0.6 & 0 & 6.0 & 10.4 \\
\hline $\begin{array}{l}\text { Patient présentant } \\
\text { une intoxication } \\
\text { alcoolo-tabagique }\end{array}$ & 7.1 & 1.9 & 76.6 & 1.3 & 0 & 0 & 4.2 & 8.8 \\
\hline $\begin{array}{l}\text { Patient atteint } \\
\text { d'une pathologie } \\
\text { cardiovasculaire }\end{array}$ & 6.5 & 1.6 & 71.1 & 2.6 & 3.2 & 0 & 6.5 & 8.4 \\
\hline $\begin{array}{l}\text { Patient sous } \\
\text { traitement } \\
\text { psychotrope }\end{array}$ & 6.5 & 1.3 & 74.4 & 1.6 & 0.3 & 0 & 5.5 & 10.4 \\
\hline Handicapé mental & 6.5 & 1.0 & 71.8 & 1.9 & 0.3 & 0 & 4.1 & 14.6 \\
\hline
\end{tabular}

* Autres combinaisons de molécules utilisées par moins de $2 \%$ des praticiens.

* Other combinations or molecules used in less than $2 \%$.

Tableau 2 : Praticiens utilisant la même molécule anesthésique pour l'adulte sain et pour les situations physiologiques et pathologiques particulières (pourcentages et intervalle de confiance).

Practitioners using the same anesthetic molecule for healthy adults and others physiological and pathological situations (percentage of responses and confidence interval).

\begin{tabular}{l|c|c|} 
& $\begin{array}{c}\text { Praticiens utilisant } \\
\text { la même molécule }\end{array}$ & $\begin{array}{c}\text { Intervalle de confiance } \\
95 \%\end{array}$ \\
\hline Enfant et adulte sains & $92.4 \%$ & {$[88.7-95.2]$} \\
\hline Femme enceinte et adulte sain & $85.5 \%$ & {$[80.8-89.4]$} \\
\hline Adulte sain et patient atteint d'intoxication alcoolo-tabagique & $92.4 \%$ & {$[88.7-95.2]$} \\
\hline Adulte sain et patient atteint d'une pathologie cardiovasculaire & $84 \%$ & {$[79.2-88.1]$} \\
\hline Adulte sain et sous traitement psychotrope & $88.4 \%$ & {$[84.0-91.9]$} \\
\hline Adulte sain et handicapé mental & $92 \%$ & {$[88.0-95.0]$}
\end{tabular}


que $92.4 \%(266 / 288=92.4 \%[88.7$ - 95.2]) des praticiens ont recours à la même molécule anesthésique pour anesthésier un enfant ou un adulte. $85.5 \%(236 / 276=85.5 \%$ [80.8 - 89.4]) utilisent la même molécule pour une femme enceinte. $92.4 \%(266 / 288=92.4 \%$ [88.7 - 95.2]) des praticiens ont recours à la même molécule (l'articaïne dans $79 \%$ des cas) pour un adulte ou un patient atteint d'une intoxication alcoolo-tabagique. Ils sont $84 \%(236 / 281=84.0 \%$ [79.2 - 88.1]) à choisir la même molécule (l'articaïne dans $73 \%$ des cas) pour un patient sain ou présentant une pathologie cardiovasculaire. $88.4 \%(244 / 276=$ $88.4 \%$ [84.0 - 91.9]) des praticiens ne modifient pas le choix de la molécule anesthésique, que le patient soit sous traitement psychotrope ou pas et $92 \%(240 / 261=92.0 \%$ [88.0 - 95.0]) que le patient soit ou non un handicapé mental.

\section{Choix du vasoconstricteur}

Lorsque l'anesthésie est réalisée chez un patient adulte sain, l'adrénaline est le vasoconstricteur préférentiellement choisi par les praticiens $(85.6 \%)(249 / 291=85.6 \%$ [81.0 - 89.4]), $10.3 \%$
(30/291 = $10.3 \%$ [7.1-14.4]) d'entre eux préfèrent la noradrénaline et $3.8 \%(11 / 291=3.8[1.9$ - 6.7]) utilisent couramment les deux molécules. La corbadrine n'est que rarement mentionnée (0.3\%). Le tableau 3 montre le choix des vasoconstricteurs pour les différentes situations physiologiques et pathologiques rencontrées chez les patients.

Pour la majorité des praticiens, le vasoconstricteur utilisé reste le même, que l'anesthésie porte sur un patient adulte sain ou présentant une situation particulière citée dans le questionnaire. $96.5 \%(279 / 289=96.5 \%$ [93.7 - 98.3]) des praticiens ne modifient pas leur choix, que le patient soit adulte ou enfant, $96 \%(262 / 273=96.0 \%$ [92.9 - 98.0]) que le patient soit âgé ou non, $91 \%$ $(213 / 234=91.0 \%$ [86.6 - 94.4]) qu'il s'agisse d'une femme enceinte ou d'un adulte, $87.4 \%$ $(201 / 230=87.4 \%$ [82.4 - 91.4]) que le patient soit diabétique ou non, $86.2 \%(200 / 232=86.2 \%$ [81.1 - 90.4]) que le patient soit atteint d'une pathologie cardiovasculaire ou non, $92.9 \%$ $(221 / 238=92.9 \%$ [88.8 - 95.8]) que le patient soit ou non sous traitement psychotrope et $95.6 \%$ médecine

buccale

chirurgie

buccale

VOL. $13, N^{\circ} 4$ 2007

page 191

Tableau 3 : Choix des vasoconstricteurs par les praticiens en Alsace (France) (100 \% = 308 chirurgiens-dentistes) (pourcentage).

Choice of vasoconstrictor in dental practice in Alsace (France) $(100 \%=308$ dentists) (percentage).

\begin{tabular}{|l|c|c|c|c|c|}
\hline Patient condition & Adrénaline & Noradrénaline & Corbadrine & $\begin{array}{c}\text { Adrénaline et } \\
\text { noradrénaline }\end{array}$ & $\begin{array}{c}\text { Pas de vaso- } \\
\text { constricteurs } \\
\text { choisis }\end{array}$ \\
\hline Adulte sain & 80.8 & 9.7 & 0.3 & 3.6 & 5.5 \\
\hline Enfant sain & 79.9 & 10.1 & 1.6 & 2.9 & 5.5 \\
\hline Patient âgé & 75.3 & 10.1 & 1.0 & 2.3 & 11.3 \\
\hline Femme enceinte & 62.0 & 11.4 & 1.6 & 1.0 & 24.0 \\
\hline Patient diabétique & 56.5 & 12.7 & 2.9 & 2.6 & 25.3 \\
\hline $\begin{array}{l}\text { Patient atteint } \\
\text { d'une pathologie } \\
\text { cardiovasculaire }\end{array}$ & 58.1 & 11.7 & 2.3 & 3.2 & 24.5 \\
\hline $\begin{array}{l}\text { Patient sous traitement } \\
\text { psychotrope }\end{array}$ & 64.6 & 8.1 & 2.3 & 1.9 & 22.7 \\
\hline Handicapé mental & 69.8 & 8.4 & 1.6 & 2.3 & 17.5 \\
\hline
\end{tabular}


Tableau 4 : Praticiens ayant recours au même vasoconstricteur pour l'adulte sain et pour les situations physiologiques et pathologiques particulières (pourcentages et indices de confiance).

Practitioners using the same vasoconstrictor for healthy adults and other conditions (percentage of responses and confidence index).

\begin{tabular}{l|cc} 
& \multicolumn{1}{|c}{$\begin{array}{c}\text { Praticiens utilisant } \\
\text { la même vasoconstricteur }\end{array}$} & $\begin{array}{c}\text { Intervalle de confiance } \\
95 \%\end{array}$ \\
\hline Enfant et adulte sains & $96.5 \%$ & {$[93.7-98.3]$} \\
\hline Patient âgé et adulte sain & $96 \%$ & {$[92.9-98.0]$} \\
\hline Femme enceinte et adulte sain & $91 \%$ & {$[86.6-94.4]$} \\
\hline $\begin{array}{l}\text { Patient diabétique et adulte sain } \\
\text { Adulte sain et patient atteint } \\
\text { d'une pathologie cardiovasculaire }\end{array}$ & $87.4 \%$ & {$[81.1-90.4]$} \\
Patient sous traitement psychotrope ou non & $86.2 \%$ & {$[88.8-95.8]$}
\end{tabular}

médecine

buccale

chirurgie

buccale

VOL. $13, \mathrm{~N}^{\circ} 4$

2007

page 192
$(241 / 252=95.6 \%$ [92.3 - 97.8]) que le patient soit handicapé mental ou non. Toutefois, entre 23 à $25 \%$ des praticiens s'abstiennent de choisir un vasoconstricteur dans certaines des situations proposées: femme enceinte, patient diabétique, patient atteint de pathologie cardiovasculaire ou sous traitement psychotrope.

Le tableau 4 expose le pourcentage des praticiens qui utilisent le même vasoconstricteur chez les patients adultes sains et chez les patients présentant les différentes situations proposées dans le questionnaire.

$51.6 \%$ des praticiens ont recours à l'anesthésie transcorticale et la majorité d'entre-eux (76.7 \%) utilisent un anesthésique contenant un vasoconstricteur pour ce type d'anesthésie.

\section{Procédures et techniques}

L'application d'un anesthétique topique a été rapporté par $59.9 \%(176 / 294=59.9 \%$ [54.0 - 65.5]) des praticiens.

$53.9 \%(159 / 295=53.9 \%[48.0-59.7])$ des praticiens ont recours à la technique d'anesthésie transcorticale, $76.7 \%(122 / 159=76.7 \%$ [69.4 -
83.1]) avec des vasoconstricteurs ; $44.7 \%$ $(71 / 159=44.7 \%$ [36.8 - 52.7]) utilisent du matériel d'injection spécifique.

Le tableau 5 montre les différentes techniques d'anesthésies pratiquées en fonction des types d'actes à réaliser et les secteurs mandibulaires et maxillaires. Dans les secteurs maxillaires et mandibulaires antérieurs, pour réaliser des soins et des actes chirurgicaux, le technique para-apicale est le technique prédominante (environ $80 \%$ ), suivie de l'intraligamentaire (entre 61 et $54 \%$ ), de la locorégionale (entre 26 et $42 \%$ ) et de la transcorticale (entre $32 \%$ et $26 \%$ ). Dans les secteurs postérieurs mandibulaires, le choix des praticiens entre ces différentes techniques est équitablement réparti. Dans tous les secteurs, pour la pose d'implants, les praticiens déclarent réaliser principalement des anesthésies para-apicales (entre $50 \%$ et $71 \%$ en fonction des secteurs), suivi de la combinaison des techniques locorégionales et para-apicales et (entre $12 \%$ et $19 \%$ ) et de la combinaison des techniques para-apicales et transcorticales (entre $2,6 \%$ et $11 \%$ ). 
Tableau 5 : Techniques anesthésiques pratiquées dans les différents secteurs en fonction des actes en Alsace (France) (100 \% = 308 chirurgiens-dentistes) (pourcentages de réponses ; plusieurs techniques anesthésiques pouvaient être choisies).

Anaesthetic techniques in various procedures in dental practice in Alsace (France) (100\% = 308 dentists) (percentage of responses; several anaesthetic techniques can be chosen).

\begin{tabular}{|c|c|c|c|c|c|}
\hline \multirow[b]{2}{*}{ Secteur } & \multirow[b]{2}{*}{ Type d'acte } & \multicolumn{4}{|c|}{ Techniques anesthésiques utilisées } \\
\hline & & Para-apicale & Locorégionale & $\begin{array}{c}\text { Intra- } \\
\text { ligamentaire }\end{array}$ & Transcorticale \\
\hline \multirow[t]{3}{*}{$\begin{array}{l}\text { Antérieur } \\
\text { mandibulaire }\end{array}$} & Soins & 79.9 & 25.6 & 61.4 & 31.8 \\
\hline & Chirurgie & 83.1 & 42.2 & 53.9 & 26.3 \\
\hline & $\begin{array}{c}\text { Chirurgie } \\
\text { implantaire (1) }\end{array}$ & 80.5 & 18.3 & 3.4 & 10.0 \\
\hline \multirow[t]{3}{*}{$\begin{array}{c}\text { Molaire } \\
\text { mandibulaire }\end{array}$} & Soins & 40.6 & 57.8 & 72.1 & 41.6 \\
\hline & Chirurgie & 56.5 & 70.1 & 59.4 & 31.2 \\
\hline & $\begin{array}{c}\text { Chirurgie } \\
\text { implantaire (2) }\end{array}$ & 71.5 & 26.4 & 5.4 & 12.2 \\
\hline \multirow[t]{3}{*}{ Maxillaires } & Soins & 92.5 & 14.3 & 24.7 & 12.3 \\
\hline & Chirurgie & 92.5 & 21.8 & 29.5 & 11.0 \\
\hline & $\begin{array}{c}\text { Chirurgie } \\
\text { implantaire (3) }\end{array}$ & 80.8 & 14.9 & 6.4 & 5.3 \\
\hline
\end{tabular}

\begin{tabular}{l}
$\begin{array}{l}\text { médecine } \\
\text { buccale } \\
\text { chirurgie } \\
\text { buccale }\end{array}$ \\
\hline VOL. $13, N^{\circ} 4$ \\
2007 \\
\hline page 193
\end{tabular}

(1) $23.7 \%$ des praticiens déclarent poser des implants dans ce secteur ; \% de réponses rapportés à ces praticiens.

$23.7 \%$ of dentists declared to place implants in this sector; \% of techniques reported to them only.

(2) $24 \%$ des praticiens déclarent poser des implants dans ce secteur ; \% de réponses rapportés à ces praticiens.

$24 \%$ of dentists declared to place implants in this sector; \% of techniques reported to them only.

(3) $24.7 \%$ des praticiens déclarent poser des implants dans ce secteur ; \% de réponses rapportés à ces praticiens.

$24.7 \%$ of dentists declared to place implants in the maxilla; \% of techniques reported to them only.

\section{Complications}

Il était demandé aux praticiens de cocher, parmi une liste de complications, celles auxquelles ils avaient étés confrontés au moins une fois au cours de leur exercice. Les réponses citées ont été, par ordre décroissant : les hématomes (93.8 \%) $(271 / 289=93.8 \%$ [90.3 - 96.3]), les réactions vagales $(70.3 \%)(206 / 293=70.3 \%$ [64.7 $75.5])$, la morsure du patient (67.6\%) (198/293=67.6 \% [61.9 - 72.9]), la nécrose du septum (42\%) 123/293 = $42.0 \%$ [36.3 - 47.9], l'is- chémie locale (23.2 \%) (68/293=23.2 \% [18.5 28.5]), les complications neurologiques (21.2\%) $(62 / 295=21.2 \%$ [16.6 - 26.3]), l'injection intravasculaire $(15 \%)(44 / 293=15 \%$ [11.1 - 19.6]), la réaction allergique $(9.9 \%)(29 / 293=9.9 \%$ [6.7 13.9], la fracture de l'aiguille (7.5 \%) (22/294 $=7.5 \%[4.7-11.1])$, la lésion nerveuse $(7.2 \%)$ (21/293 = $7.2 \%$ [4.5- 10.7]) et les complications cardiovasculaires. $(5.1 \%)(15 / 293=5.1 \%$ [2.9 8.3]). 
médecine buccale chirurgie buccale

VOL. $13, \mathrm{~N}^{\circ} 4$ 2007

page 194
Tableau 6 : Complications rapportées par les praticiens * (pourcentages). Complications reported by surveyed by practitioners* (percentage).

\begin{tabular}{|c|c|c|}
\hline Complications & Pourcentage des praticiens & Intervalle de confiance 95\% \\
\hline Hématome local & $93.8 \%$ & {$[90.3-96.3]$} \\
\hline Réaction vagale & $70.3 \%$ & {$[64.7-75.5]$} \\
\hline Morsure & $67.6 \%$ & {$[61.9-72.9]$} \\
\hline Nécrose du septum & $42 \%$ & {$[36.3-47.9]$} \\
\hline Ischémie locale & $23.2 \%$ & {$[18.5-28.5]$} \\
\hline Réaction neurologique & $21.2 \%$ & {$[16.6-26.3]$} \\
\hline Injection intra-vasculaire & $15 \%$ & {$[11.1-19.6]$} \\
\hline Réaction allergique & $9.9 \%$ & {$[6.7-13.9]$} \\
\hline Bris de l'aiguille & $7.5 \%$ & {$[4.7-11.1]$} \\
\hline Lésion nerveuse & $7.2 \%$ & {$[4.5-10.7]$} \\
\hline Complication cardiovasculaire & $5.1 \%$ & {$[2.9-8.3]$} \\
\hline
\end{tabular}

* Pourcentage de praticiens ayant été confronté à ces complications au moins une fois.

* Proportion of practitioners who have reported to have experienced the stated complication at least once.

\section{DISCUSSION}

Les résultats de cette enquête décrivent probablement avec justesse les pratiques courantes dans la prise en charge de la douleur en odontologie en Alsace. Les praticiens ayant répondu présentent toutes les durées d'expérience professionnelle et les différents modes d'exercice existant ainsi que la pratique dans les zones urbaines ou non urbaines.

Le choix très largement majoritaire de l'articaïne parmi les molécules anesthésiques conduit à un certain nombre de commentaires. L'articaïne est une des dernières molécules disponibles pour la prise en charge de la douleur dans la pratique odontologique [5]. Son délai d'action rapide et sa puissance ( 1.5 fois celle de la lidocaïne, 1.9 fois celle de la procaïne) ${ }^{[6,7,5,8]}$. Sa durée d'action est modérée, ce qui peut éventuellement limiter son bénéfice lors des actes particulièrement longs comme certains traitements endodontiques pour lesquels les molécules anesthésiques de longue durée d'action peuvent se révéler plus pratiques (comme la prilocaïne). L'articaïne est considéré comme la molécule anesthésique indiquée pour l'anesthésie de la femme enceinte [6]. Du fait de son métabolisme hépatique et de ses propriétés déprimantes pour le myocarde, elle doit être utilisée avec prudence chez les patients souffrants de pathologies hépatiques ou ceux atteints d'insuffisance cardiaque ${ }^{[8]}$. Toutefois, elle est considérée comme étant la molecule anesthésique la moins toxique ${ }^{[5,8]}$.

Une enquête ${ }^{[1]}$ menée précédemment en France, partant sur 2000 chirurgiens-dentistes (354 réponses) a montré que $87.2 \%$ des praticiens ayant répondu utilisaient l'articaïne. Seulement $12.8 \%$ des praticiens avaient recours à la lidocaiine et $11.8 \%$ à la mépivacaïne. A l'époque où a été réalisée cette enquête (1988), l'articaïne était déjà la molécule anesthésique préférée des praticiens français avec la lidocaïne comme alterna- 
tive. Dans une enquête récemment conduite et publiée au Royaume-Uni [2], l'articaïne ne constituait que le troisième choix des praticiens derrière la lidocaïne et la prilocaïne. Il est intéressant de constater que ce choix variait significativement chez les praticiens présentant une expérience professionnelle supérieure ou inférieure à 5 ans [2] . Le principal argument avancé pour justifier le choix de la lidocaïne était son efficacité (74\%) [2]. En Allemagne, comme le rapporte Daublander et coll. [3], l'articaïne est la molécule majoritairement utilisée ( $91 \%$ des cas.) La lidocaïne n'est utilisée que dans $2 \%$ des cas (essentiellement chez les patients sains) et la mépivacaïne dans $3 \%$ des cas (sans vasoconstricteurs, principalement chez les patients présentant des conditions cardiovasculaires particulières). Plus récemment, au Brésil [4], Ramacciato et coll. rapportent dans une enquête limitée à 133 praticiens que la prilocaïne est la molécule anesthésique la plus couramment employée (86 \%), choisie sur l'argument de son efficacité.

Selon les données de notre étude, la plupart des praticiens $(74.7 \%)(230 / 290=79.3$ [74.2 - 83.8]) ne possèdent qu'une molécule anesthésique dans leur cabinet, ce qui limite la capacité à modifier leur choix concernant la molécule anesthésique en fonction des facteurs de risque du patient.

Dans notre enquête, la lidocaïne était la seconde molécule la plus fréquemment utilisée alors qu'elle est la plus couramment employée au RoyaumeUni ${ }^{[2]}$. La lidocaïne était la deuxième molécule à avoir été utilisée pour les anesthésies locales en odontologie. Elle a probablement le plus grand nombre de données confirmant sa sécurité d'utilisation, ce qui lui confère à son statut de molécule de référence. Elle a un délai d'action, une puissance et une durée d'action moyennes [6]. Chez la femme enceinte, la concentration plasmatique fœtale correspond à $40 \%$ de la concentration plasmatique maternelle. Elle est métabolisée par le foie et ses métabolites sont éliminés par le rein. La toxicité de la lidocaïne est faible aux doses utilisées en odontologie.

La mépivacaïne est très rarement utilisée en dépit de ses propriétés. Elle possède une action de vasoconstriction intrinsèque ${ }^{[9]}$ qui permet d'augmenter sa durée d'action, ce qui se révèle parti- culièrement utile lorsque l'adrénaline ou la noradrénaline sont contre-indiquées. Elle est contreindiquée chez la femme enceinte du fait de son transfert au fœtus qui n'est pas capable de métaboliser la mépivacaïne. Ses autres propriétés sont similaires à celles de la lidocaïne.

La prilocaïne n'a été mentionnée que par deux praticiens dans notre enquête. Son avantage réside dans sa longue durée d'action. Elle est contre-indiquée chez la femme enceinte en raison des rares cas de méthémoglobinémie chez le fœtus [10,11]. Elle est plus fréquemment utilisée au Royaume-Uni, spécialement chez les patients atteints de pathologies cardiovasculaires où elle est alors combinée avec de la félypressine comme vasoconstricteur.

Contrairement aux molécules précédentes qui étaient des molécules de la famille des aminoamides, la procaïne est un amino-ester. Elle n'a pas été citée dans notre enquête, bien qu'elle soit indiquée chez les patients atteints de dysfonctionnements hépatiques car elle est métabolisée dans le plasma en acide para-amino-benzoïque [9] . Par contre, l'acide para-amino-benzoïque entraine souvent des réactions d'hypersensibilité et des réactions allergiques ${ }^{[11]}$. Comme sa durée d'action est courte, la procaïne est toujours utilisée avec un vasoconstricteur.

Dans notre enquête, il y a peu de différence dans le choix des molécules (anesthésiques ou vasoconstricteurs) par les praticiens en fonction de la situation physiologique ou pathologique du patient. La seule modification significative était l'augmentation des abstentions de choix de vasoconstricteurs de $4 \%$ à plus de $10 \%$ pour les anesthésies concernant les femmes enceintes et les patients handicapés mentaux ou sous traitement psychotrope. Lors de la grossesse, les praticiens préfèrent souvent repousser les soins jusqu'à l'accouchement. Les motivations sont moins évidentes pour expliquer l'absence de réponse pour les deux autres catégories de situation particulières.

Quand on analyse le choix des vasoconstricteurs, on s'aperçoit que la grande majorité des praticiens alsaciens préfèrent les utiliser (85.6\%) $(249 / 291=85.6 \%$ [81.0 - 89.4]). Ce choix est très similaire au Royaume-Uni [2] et en Allemagne [3]. médecine

buccale

chirurgie

buccale

VOL. $13, N^{\circ} 4$ 2007

page 195 
médecine

buccale

chirurgie

buccale

VOL. $13, \mathrm{~N}^{\circ} 4$ 2007

page 196
La noradrénaline est très peu utilisée. Le choix du vasoconstricteur n'est pas modifié dans les cas de situations physiopathologiques particulières sauf pour une exception : pour anesthésier un patient diabétique, le pourcentage de praticiens choisissant des carpules contenant de la noradrénaline augmente (de $9.7 \%$ à $12.7 \%$ ). Il est admis que pour les patients diabétiques, l'usage de noradrénaline est indiqué en raison de l'effet hyperglycémiant de l'adrénaline. L'abstention du choix du vasoconstricteur augmente de façon significative (abstention comprise entre 17.5 et $25.3 \%$ suivant les situations) lorsque la question du choix du vasoconstricteur porte sur une anesthésie chez une femme enceinte, un patient diabétique, un handicapé mental ou un patient sous traitement psychotrope. Lorsque la question porte sur l'anesthésie chez un patient âgé, l'absence de choix de vasoconstricteur augmente légèrement $(11.3 \%$ contre $5.5 \%$ chez les patients sains).

Ces résultats sont similaires à ceux obtenus au Royaume-Uni [2], où l'adrénaline est toutefois remplacée par la félypressine (dérivée de la vasopressine) dans certaines conditions physiopathologiques particulières (en association avec la prilocaïne.) Au Royaume-Uni, $20 \%$ des praticiens interrogés choisissent l'utilisation de carpules ne contenant pas de vasoconstricteur essentiellement pour l'anesthésie de patients ayant eu récemment un infarctus du myocarde (moins de 6 mois) ${ }^{[2]}$. Cette enquête apprend également que les patients atteints de diabète déséquilibré sont anesthésiés sans vasoconstricteurs. Dans les résultats de l'enquête allemande [3], l'utilisation de mépivacaïne augmente significativement lorsque l'anesthésie concerne un patient atteint de pathologie cardiovasculaire.

La précédente enquête française ${ }^{[1]}$ précise que seulement $48.5 \%$ des praticiens ont recours systématiquement à des solutions anesthésiques contenant un vasoconstricteur sans que les molécules utilisées soient spécifiées.

Les résultats de notre enquête confirment que $59.9 \%(176 / 294=59.9 \%$ [54.0 - 65.5]) des praticiens alsaciens ont recours à une pré-anesthésie topique avant l'infiltration. Pour mémoire, dans la précédente enquête menée en France, 78,7 \% des praticiens [1] utilisaient un anesthésique topique. Ce pourcentage inférieur ne traduit pas forcément une diminution de l'utilisation d'anesthésique topique : il semble plutôt être dû à l'hétérogénéité des populations interrogées qu'au facteur temps. Toutefois, nous nous attendions à ce que l'usage de l'anesthésique topique soit plus répandu non seulement pour le confort qu'elle apporte aux patients lors de l'infiltration, mais aussi pour la désinfection du site d'injection que cette application assure.

Dans notre enquête, l'étude des choix des praticiens concernant les techniques utilisées démontre que $53.9 \%(159 / 295=53.9 \%$ [48.0 59.7]) ont recours à l'anesthésie transcorticale et que, dans la majorité des cas, cette technique se pratiquait avec des carpules contenant un vasoconstricteur. Pourtant, la survenue de nécroses du septum (rare complication due à une ischémie vasculaire locale) a été attribuée, entre autres, à la présence de vasoconstricteurs dans les solutions injectées avec cette technique.

Quels que soient les secteurs et le type d'acte envisagé (soins, chirurgie ou pose d'implants), l'anesthésie para-apicale représente la technique la plus couramment employée, occasionnellement complétée par une anesthésie intra-ligamentaire ou éventuellement une anesthésie locorégionale. Le choix de la technique d'anesthésie lors de la pose d'implants ne varie pas significativement en fonction des différents secteurs.

La plupart des praticiens ont rapporté avoir été confrontés au moins une fois à une complication, le plus souvent locale. Les plus fréquemment citées sont, par ordre décroissant : I'hématome, le malaise vagal, la morsure du patient et la nécrose du septum. Les autres complications citées dans le questionnaire ont été très peu relatées par les praticiens. Dans l'enquête brésilienne ${ }^{[4]}, 27 \%$ des praticiens ont rencontré une complication locale, la plus fréquente était l'œdème ; seulement $3.7 \%$ des praticiens rapportent avoir été confrontés à une complication systémique.

L'étude allemande [3] était plus spécifiquement axée sur l'incidence des complications rapportées : $4.5 \%$ des 2731 patients ont présenté une 
complication après l'anesthésie locale. Les plus fréquentes étaient : un malaise (1.3\%), une tachycardie (1.1\%), une agitation (1.1\%), des nausées $(0.8 \%)$ et des tremblements $(0.7 \%)$. Lorsque le patient a une anamnèse médicale comportant des facteurs de risques, l'incidence des complications augmente (5.7\%). Il est toutefois intéressent de noter que parmi les 600 patients de cette étude atteints d'une pathologie cardiovasculaire, aucune complication sévère n'a été rapportée.

La précédente enquête française ${ }^{[1]}$ rapporte des complications locales bénignes (ulcération, douleurs au point d'injection). Les réactions vagotoniques constituaient les plus fréquentes complications systémiques rapportées [1].

Au total, notre enquête, comme les précédentes, rapportent majoritairement diverses complications bénignes.

\section{CONCLUSIONS}

Cette enquête a été la première menée auprès de tous les praticiens exerçant dans une large région française, l'Alsace, avec pour but de décrire les usages et les pratiques habituelles en anesthésiologie en odontologie.

Lorsque le questionnaire concerne l'utilisation des molécules anesthésiques, les trois-quarts des praticiens répondent n'avoir recours qu'à une seule molécule anesthésique (l'articaïne). La lidocaïne constitue, dans une bien moindre mesure, le second choix. Le choix de la molécule anesthésique n'est que rarement influencé par les conditions physiopathologiques du patient. Parmi les trois vasoconstricteurs proposés dans le questionnaire, l'adrénaline est le plus fréquemment utilisée par les praticiens, suivi par la noradrénaline. De manière générale, lorsque la question du choix du vasoconstricteur se rapporte à un patient présentant des conditions physiopathologiques particulières, environ $25 \%$ des praticiens ne choisissent aucun des vasoconstricteurs dans la liste proposée par le questionnaire

Bien que les molécules anesthésiques présentent différentes propriétés dont la spécificité pourrait être exploitée pour les patients présentant des conditions physiopathologiques particulières, le fait que les praticiens ne possèdent qu'une ou éventuellement deux molécules anesthésiques ne permet pas aux praticiens de pratiquer une anesthésie différente lorsque les patients présentent des conditions particulières. Le choix de l' " anesthésique unique » est favorisé par la grande sécurité d'utilisation de l'articaïne. La confiance et l'expérience clinique des praticiens les encouragent à ne se servir que de cette seule molécule.

La technique d'anesthésie para-apicale est la plus pratiquée dans tous les secteurs, quel que soit le type d'acte envisagé. Environ la moitié des praticiens ont recours à l'anesthésie transcorticale. Dans les secteurs antérieurs mandibulaires, l'anesthésie para-apicale est largement la plus utilisée, dans une moindre mesure, les anesthésies intraligamentaires et les anesthésies locorégionales sont également pratiquées. Par contre, peu de praticiens relatent l'utilisation de l'anesthésie transcorticale dans ces secteurs. Dans les secteurs molaires mandibulaires, toutes les techniques sont utilisées bien qu'un quart des praticiens affirment ne pas réaliser d'anesthésie locorégionale. Au maxillaire, l'anesthésie paraapicale est la technique la plus pratiquée bien qu'environ un cinquième des praticiens combinent l'usage de l'anesthésie locorégionale et intraligamentaire ; l'anesthésie transcorticale est peu pratiquée dans ces secteurs.

Un tiers des praticiens ayant répondu déclare exercer la chirurgie implantaire. En général, ils utilisent l'anesthésie para-apicale, rarement combinée à l'anesthésie locorégionale. Les hématomes, les malaises vagaux, les morsures et les nécroses du septum sont les complications locales bénignes le plus fréquemment rapportées par les praticiens. médecine

buccale

chirurgie

buccale

VOL. $13, N^{\circ} 4$ 2007

page 197 


\section{RÉFÉRENCES}

1 - Nasseripour N, Pham TTH, Arreto CD, Wierzba CB. Anesthésie dentaire au quotidien. Enquête auprès des praticiens français. Actual Odonto-Stomatol 2000 ; 210 : 221-36.

2 - Corbett IP, Ramacciato JC, Groppo FC, Meechan JG. A survey of local anaesthetic use among general dental practitioners in the UK attending postgraduate courses on pain control. Br Dent J 2005 ; 199 : 784-7 (discussion 778).

3 - Daublander M, Muller R, Lipp MDW. The incidence of complications associated with local anesthesia in dentistry. Anesth Prog 1997, 44 : 132-41.

4 - Ramacciato JC, Ranali J, Volpato MC, Groppo FC, Florio FM, Soarez PCO. Local anesthetics use by dentists profile. J Dent Res 2002 ; 81 (Spec issue B) : 491.

5 - Malamed S. Nouveaux anesthésiques. Actual OdontoStomatol $1991 ; 176$ : 561-9. (Original : Newly available anesthetic formulations. Rev Belge Dent 2000 ; 55 : 918).
6 - Guerin T, Maman L., Wierzba CB. Mise au point sur les anesthésiques locaux injectables en 1997. Chir Dent France 1997 ; 848 : 23-32.

7 - Lendais I, Limoge A. Choix d'un anesthésique local. Etude comparative de quatre molécules. Chir Dent France 1987 ; $368: 1-7$.

8 - Vree TB, Gielen MJ. Clinical pharmacology and the use of articaine for local and regional anesthesia. Best Pract Res Clin Anaesthesiol 2005 ; 19 : 293-308

9 - Cavaillon JP, Haag R. L'anesthésie locale et loco-régionale (pp 181-212). In Manuel des urgences en pratique odonto-stomatologique. Cavaillon J-P, Girard P, Noto R. eds. Masson, Paris, 1988.

10 - Commissionat Y, Rimet J-L. Anesthésie locale. Complications. Actual Odonto-stomatol 1992 ; 179 : 497-505.

11 - Conseiller C, Guesnon P, Leoni J. Anesthésiques locaux ( $3^{e}$ partie) - Autres anesthésiques locaux. Sem Hôp Paris 1985 ; 61 : 2857-67. médecine

buccale

chirurgie

buccale

VOL. $13, \mathrm{~N}^{\circ} 4$ 2007 